\title{
A multiscale shape descriptor based on differential entropy
}

\author{
Raphael G. Pinheiro* José G. F. Lopes* Allan C. Carneiro** \\ Marcelo M. S. de Souza ${ }^{* * *}$ Fátima N. S. Medeiros* \\ * Universidade Federal do Ceará, Campus do Pici, Fortaleza, CE, \\ Brazil (e-mail: raphaelgomespinheiro@hotmail.com, \\ gerardofonteles@gmail.com,fsombra@ufc.br). \\ ** Instituto Federal de Educação, Ciência e Tecnologia do Ceará, CE, \\ Brazil (e-mail: allan.carneiro@ifce.edu.br). \\ *** Universidade Federal do Ceará, Campus de Sobral, Sobral, CE, \\ Brazil (e-mail: marcelo@ufc.br).
}

\begin{abstract}
We propose a shape descriptor based on the differential entropy of multiscale curvature (MEC), which has a better shape discrimination ability than the normalised multiscale bending energy (NMBE) descriptor. We compare both NMBE and MEC with a monoscale descriptor by conducting multiple-class classification experiments on images from three public datasets: Kimia99 (99 shapes/9 classes), MPEG7-CE (1400 shapes/70 classes) and Flavia leaves (1907 shapes/32 classes). The classification results for precision, recall and F1-score measures, show that the use of MEC improved shape description for the MPEG7-CE and Flavia datasets. A qualitative analysis based on visualisation of pairwise Euclidean distance matrices also confirmed that MEC is a competitive method of shape description compared to NMBE for these datasets.
\end{abstract}

Keywords: Shape descriptor; Differential entropy; Multiscale curvature; Normalised multiscale bending energy; Leaf shapes.

\section{INTRODUCTION}

Although general purpose invariant descriptors are difficult to find, they can be effectively combined with other methods for shape-based object recognition, classification and indexing (Ullman, 1995). Shape features are stable image characteristics compared with colour and texture features and are relevant in the field of object recognition since they do not significantly change when the contrast and brightness are modified (Yang and Yu, 2019).

Over recent decades, researchers have exploited a wide range of information theory concepts to solve computer vision and pattern recognition problems (Escolano et al., 2009; Das et al., 2019) since measures such as entropy and mutual information relate directly to the information content of the signals (Singh and Príncipe, 2011). Page et al. (2003) applied the Shannon entropy of curvature to measure the shape complexity in shape analysis. Likewise, the concept of correntropy (Liu et al., 2006) and the Cauchy-Schwarz divergence have been used for shape matching (Hasanbelliu et al., 2014).

Shape analysis and recognition tasks can benefit from algorithms that use multiscale curvature to represent the shapes of objects (Paula et al., 2013; Souza et al., 2016; Carneiro et al., 2017) or monoscale description (Ushizima et al., 2015). Shape representation can also be performed to handle corners in tasks such as scene analysis, polygonal

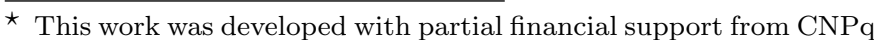
(\#306600/2016 - 1), FUNCAP and CAPES-Brazil (Finance Code 001).
}

approximation, feature matching, robot navigation, shape similarity and object tracking, to name only a few (Paula et al., 2013).

Souza et al. (2016) designed a new approach to optimising the parameters of the normalised multiscale bending energy (NMBE) (Cesar Jr and Costa, 1997) in order to improve its shape discrimination ability. Carneiro et al. (2017) investigated the role of different cost functions in the optimisation of NMBE by performing experiments on shapes from different public datasets.

In this work, we present the multiscale entropy of curvature (MEC), which is a descriptor that discriminates subtle details in the shape contour by evaluating the probability of occurrence of the feature based on curvature. Thus, this descriptor relies on the multiscale curvature in the same way as the ones proposed in (Souza et al., 2016; Carneiro et al., 2017, 2019).

Our assumption is that the differential entropy of the curvature is sensitive to the occurrence of local features on the shape contour. We assess the discrimination ability of NMBE and MEC as well as a monoscale descriptor by carrying out general purpose multiple-class shape classification experiments using three well-known public shape datasets: Kimia99, Flavia leaves and MPEG7-CE. We employ the precision and recall measures and F1-score to evaluate the shape classification results, and evaluate both descriptors qualitatively by calculating the pairwise Euclidean distance matrices for the shapes of each dataset under investigation. The main contributions of this work 
are: a) the design and testing of a new invariant shape descriptor that is relatively sensitive to local features and that relies on the differential entropy of curvature; b) a qualitative evaluation of the performance of both multiscale shape descriptors using pairwise distance matrices; and c) the proposal of a multiscale descriptor that is competitive with those in the literature for describing real shapes in computer vision applications such as leaf shape recognition. Additionally, we compare the results of both multiscale descriptors (NMBE and MEC) with a monoscale descriptor (MONO) introduced in (Ushizima et al., 2015) that combines region-based and contour-based characteristics (Zhang and Lu, 2004).

The paper is structured as follows. Section 2 describes the concepts of the Shannon entropy, differential entropy and multiscale curvature, and Section 3 introduces the MEC shape description. Section 4 presents our experiments and discusses the results. Finally, Section 5 draws our conclusions.

\section{BACKGROUND CONCEPTS}

This section introduces the concepts of the differential entropy and multiscale curvature, which are required for the our descriptor.

\subsection{Entropy}

Entropy measures the average amount of uncertainty in the outcome of a random variable (rv) (Rényi, 1961). Let the realisation of a discrete $r v X_{d}$, compounded by $N$ symbols, be a message. The discrete Shannon entropy describes the average amount of information conveyed per message, and this is defined as (Cover and Thomas, 2006):

$$
H\left(X_{d}\right)=-\sum_{k=1}^{N} p_{k} \log p_{k}
$$

where $p_{k}$ is the probability that the message symbol $x_{k}$ occurs. In the continuous case, the so called differential entropy is defined as (Cover and Thomas, 2006):

$$
h\left(X_{c}\right)=-\int_{-\infty}^{\infty} p(x) \log p(x) d x,
$$

where $p(x)$ is the probability density function (pdf) that describes the behaviour of the continuous $r v X_{c}$.

The differential entropy has useful properties that are related to the translation and scaling of an $r v$ (Cover and Thomas, 2006), which are defined as follows:

$$
h\left(X_{c}+a\right)=h\left(X_{c}\right)
$$

and

$$
h\left(a X_{c}\right)=h\left(X_{c}\right)+\log a .
$$

Equation 3 states that the translation of an $r v$ by a constant $a$ does not affect its differential entropy, while Equation 4 states that scaling of an $r v$ by a constant $a$ corresponds to translating its differential entropy by $\log a$. These properties are useful in developing an entropybased descriptor that is invariant to shape scaling and translation.

\subsection{Multiscale curvature}

The multiscale curvature (Cesar Jr. and Costa, 1996; Mokhtarian and Mackworth, 1992) is a more robust method for shape invariant description than the curvature itself. In practise, the calculation of curvature accentuates high frequency noise, and this limits its application to representing discrete contours (Costa and Cesar Jr, 2009). The multiscale curvature is a coarse-to-fine shape description that acts as a prior filter for the parametric coordinates of the shape contour, $C=(x(t), y(t))$, with a Gaussian kernel, and generates the smoothed version, $C_{\sigma}=\left(x_{\sigma}(t), y_{\sigma}(t)\right)$ at scale $\sigma$ and $t \in[0,1]$. The scale parameter controls the level of shape details, and corresponds to the standard deviation of the Gaussian kernel.

The multiscale curvature at scale $\sigma$ is given by (Costa and Cesar Jr, 2009):

$$
\kappa(\sigma, t)=\frac{\dot{x}_{\sigma}(t) \ddot{y}_{\sigma}(t)-\ddot{x}_{\sigma}(t) \dot{y}_{\sigma}(t)}{\left[\left(\dot{x}_{\sigma}(t)\right)^{2}+\left(\dot{y}_{\sigma}(t)\right)^{2}\right]^{\frac{3}{2}}},
$$

where $\left(\dot{x}_{\sigma}(t), \dot{y}_{\sigma}(t)\right)$ and $\left(\ddot{x}_{\sigma}(t), \ddot{y}_{\sigma}(t)\right)$ are the first and second order derivatives of $\left(x_{\sigma}(t), y_{\sigma}(t)\right)$, respectively.

\section{METHODOLOGY}

This section presents the proposed descriptor for shape feature extraction, based on MEC, and the pipeline used for performance evaluation via classification experiments.

\subsection{Multiscale entropy of curvature}

Our assumption in deriving MEC is that the evaluation of the probability of shape feature occurrence leads to a more sensitive multiscale descriptor. Indeed, at a given scale, the features embodied in the contour curvature of the object are likely to mould the shape of the underlying pdf of the curvature. Entropy is therefore a convenient measure for extracting the shape features encoded into the pdf of the curvature at varying scales.

In order to calculate the differential entropy of multiscale curvature, it is necessary to estimate the curvature pdf at each scale considered, and this can be done either parametrically or non-parametrically. One nonparametric approach to estimating pdfs directly from the data samples, without requiring previous assumptions about the underlying probability model, is the Parzen window method (Webb, 2002). We adopt this approach in the following.

Let $\kappa[\sigma, n]=(\kappa[\sigma, 1], \kappa[\sigma, 2], \ldots, \kappa[\sigma, N])$ be the $N$ sampled values of the multiscale curvature at scale $\sigma$. The estimated pdf of the multiscale curvature, $p_{\sigma}(\kappa)$, at scale $\sigma$ and a given curvature value $\kappa$, through the Parzen window, is given by (Webb, 2002):

$$
p_{\sigma}(\kappa)=\frac{1}{b N} \sum_{i=1}^{N} \Psi\left(\frac{\kappa-\kappa[\sigma, i]}{b}\right)
$$

where

$$
\Psi\left(\frac{\kappa-\kappa[\sigma, i]}{b}\right)=\frac{1}{\sqrt{2 \pi}} \exp \left(-\frac{(\kappa-\kappa[\sigma, i])^{2}}{2 b^{2}}\right),
$$


is a Gaussian kernel window and $b$ the kernel window bandwidth. The $b$ parameter controls the smoothing degree in the pdf estimation. This parameter is critical because excessive smoothing implies loss of information, whereas slightly smoothing leads to a noisy estimation. Aiming to find an optimum value of the $b$ parameter, Silverman and Green (1986) proposed its calculation as:

$$
b=\left(\frac{4 \hat{s}^{5}}{3 N}\right)^{\frac{1}{5}},
$$

where $\hat{s}$ is the standard deviation of the curvature samples and $N$ is the number of samples involved in the estimation.

Thus, the differential entropy of the multiscale curvature at scale $\sigma$ can be approximated by the integral:

$$
h_{\sigma}(\kappa)=-\int_{\kappa_{\min }}^{\kappa_{\max }} p_{\sigma}(\kappa) \log p_{\sigma}(\kappa) d \kappa,
$$

where a numerical integration method is employed to evaluate this integral, $E\{\cdot\}$ represents the expected value and $\kappa_{\min }=E\{\kappa[\sigma, n]\}-5 \hat{s}$ and $\kappa_{\max }=E\{\kappa[\sigma, n]\}+5 \hat{s}$.

Although the result of Equation 9 is invariant to shape translation and rotation, it is not invariant to shape scaling. However, this invariance can be attained through the properties of Equation 4. Normalizing the differential entropy with respect to the shape perimeter $\left(L_{\sigma}\right)$ yields:

$$
\hat{h}_{\sigma}(\kappa)=h_{\sigma}(\kappa)+\log L_{\sigma} .
$$

The MEC descriptor evaluates the multiscale curvature in Equations 5, 9 and 10 for a given set of discrete scales $\left(\sigma_{1} \sigma_{2} \cdots \sigma_{M}\right)$ and it is defined as:

$$
\mathrm{MEC}=\left(\hat{h}_{\sigma_{1}}(\kappa) \hat{h}_{\sigma_{2}}(\kappa) \cdots \hat{h}_{\sigma_{M}}(\kappa)\right)
$$

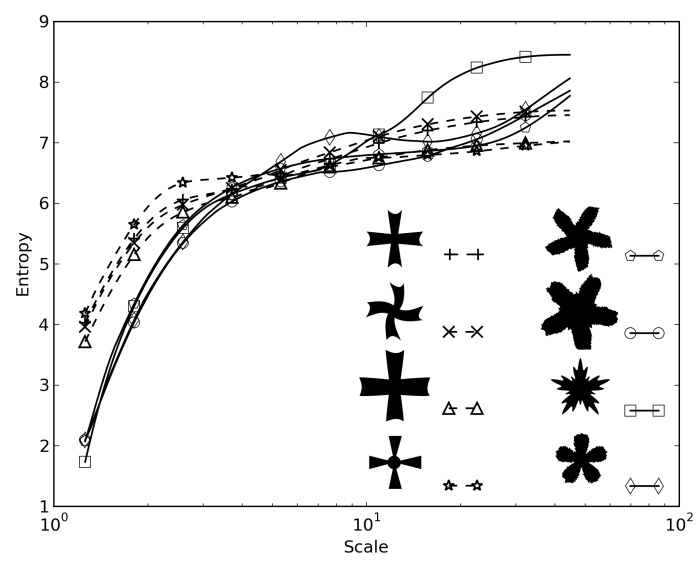

Figure 1. Shape description based on the multiscale entropy of curvature for two classes of image.

Figure 1 illustrates shapes from two classes of the MPEG7$\mathrm{CE}$ dataset and their corresponding MEC descriptions. We perform the smoothing process in the frequency domain. The lower scales correspond to a narrower bandwidth filtering, and this leads to a coarse shape representation.
Conversely, higher scales correspond to wider bandwidth filtering, and thus a fine-detailed shape representation. We observe that MEC is relatively robust to intra-class variability, as there is no significant separation between the shapes represented by the dotted lines. A similar analysis applies to the class of shapes represented by the continuous lines. In addition, Figure 1 shows that better discrimination is achieved between classes, i.e. dotted and continuous lines, at lower scales. However, at intermediate and higher scales, the representation of the two shape classes is likely to overlap when using MEC. Although giving poor class discrimination at high scales for both classes, the MEC description for these classes tends to follow different trends with the increase in scale. It is worth mentioning that at higher scales, our descriptor is able to reveal intra-class variability, as shown by the last two fivepetal shapes.

In order to evaluate and compare the proposed methodology for feature extraction, we perform classification experiments on synthetic and real images described by MEC, NMBE and a monoscale descriptor.

Figure 2 shows the pipeline employed in these experiments. The feature extraction step represents the multiscale shape description, i.e. NMBE and MEC, and a monoscale description used to extract features from the image datasets under test. Following the recommendation in (Cesar $\mathrm{Jr}$ and Costa, 1997), we set five scales $(\sigma)$ for both NMBE and MEC that varied from 0.4 to 100 , yielding an $M \times 5$ matrix, where $M$ is the number of shape samples in the dataset.

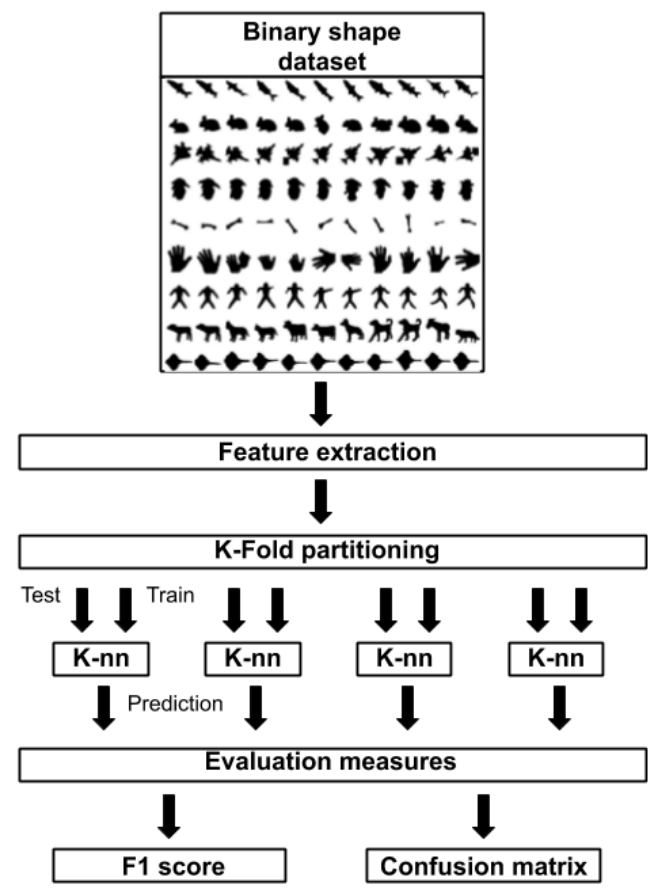

Figure 2. Cross validation experiment conducted to evaluate the performance of MEC in shape classification.

Here, we adopted the K-Fold with four partitions and the K-nearest neighbours (K-nn, $K=3$ ) classifier. Since each matrix line corresponds to a distinct shape descriptor, the shapes were randomly separated into $N$ folds for training 
and testing in the classification experiments. The number of folds $N$ was adjusted to give standard deviation values smaller than 0.04 for the average F1-score. This metric is defined as the harmonic mean of precision $(P)$ and recall $(R)$ :

$$
\text { F1-score }=\frac{P \times R}{P+R}
$$

where

$$
P=\frac{t p}{t p+f p}
$$

and

$$
R=\frac{t p}{t p+f n}
$$

For a given class, $t p$ (true positive) represents the number of shapes correctly labelled by the classifier as belonging to a particular class. $f p$ (false positive) is the number of shapes labelled as belonging to a class to which they do not belong, and $f n$ (false negative) corresponds to the number of shapes that belong to a particular class but were labelled as belonging to another.

\section{EXPERIMENTAL RESULTS}

This section presents the qualitative and quantitative evaluation of both multiscale descriptors and a monoscale descriptor. MONO comprises the circularity, aspect ratio, roundness and solidity attributes and Ushizima et al. (2015) have applied it to images from the NLM Pills dataset for feature extraction and clustering.

\subsection{Qualitative evaluation}

The greyscale images in Figure 3a and Figure 3b represent the pairwise Euclidean distance matrices of MEC for different classes of shapes from the Kimia99 dataset. The dark shaded areas in these matrices refer to the smallest distances, while the lighter areas correspond to the largest ones. These images allow for the evaluation of the discrimination ability of the MEC shape descriptor.

Figure 3a shows that the distances between shape descriptors belonging to the same class are smaller than those between shape descriptors from distinct classes. MEC therefore provides low intra-class variability (presence of dark squares) and high inter-class separability for the shape classes under analysis. However, Figure 3a also demonstrates that MEC was not able to discriminate the shapes representing the rabbit and alien classes, shown in Figure $3 \mathrm{a}$ as classes 2 and 4, respectively. The square regions comprising the intersection of these classes (line 2 column 4 , and line 4 column 2) are predominantly dark, indicating that the Euclidean distances between the shapes in these distinct classes are small; except for the fine details, the shapes in both classes are relatively similar. The descriptor was not sensitive to the fine local details of these shapes, and identified them as being similar.

Differences between the images in the classes of hands, humans and four-legged animals or quadrupeds (classes 2, 3 and 4) are not evident in Figure 3b. We conclude that this matrix cannot handle the variability within these classes of shapes. However, MEC effectively discriminated objects from the tool and ray classes (classes 1 and 5).

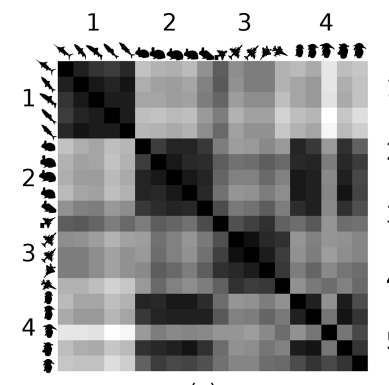

(a)

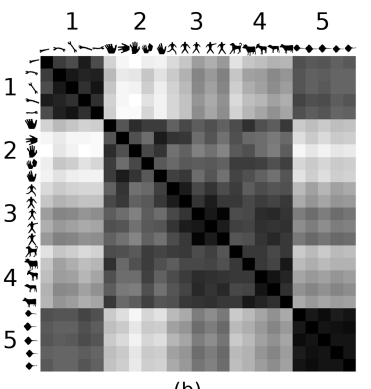

(b)
Figure 3. Greyscale images corresponding to the pairwise Euclidean distances between MECs for different classes of shapes. The shaded areas correspond to small distances between shapes, whereas the lighter areas indicate large distances.

\subsection{Classification experiments}

Table 1 summarises our classification results for three datasets. The performance of both multiscale shape descriptors was relatively similar for the Kimia99 and Flavia datasets. However, the results with shapes from Kimia99 show that MONO enhanced the precision and recall values in 4\%, approximately. Moreover, MONO was able to discriminate shapes from Kimia99 due to the high interclass variability and the low intra-class variability of this dataset. The good performance of MONO to deal with shape classes from Kimia99 was due to local geometric aspects of these shapes that enabled it to discriminate them well, as Figure 4a illustrates. On the other hand, the monoscale descriptor was not able to discriminate classes of shapes from the Flavia dataset. As a matter of fact, the MONO descriptor relies on local geometrical shape features which are similar for leaves of distinct plant specimens (low inter-class variability). In addition, such geometrical attributes may vary significantly for leaves from the same plant specimen (high intra-class variability).

The MEC descriptor improved the F1-score value of the NMBE descriptor by about $5 \%$ for the Flavia dataset. Both multiscale descriptors failed to perform adequately with the MPEG7-CE dataset, which is well-known to be a challenging dataset for shape retrieval and classification tasks. However, the MEC descriptor outperformed NMBE for this particular dataset. In addition, we observed that the precision and recall values tended to decrease as the number of classes and shapes in the dataset increased. Figure 4a shows that both descriptors performed similarly in characterising shapes from the Kimia99 dataset, and this is confirmed by the classification results in Table 1 . MEC was not able to discriminate the shapes in the alien and rabbit classes, nor those from the bone and fish classes. Figure $4 \mathrm{~b}$ shows that NMBE performed marginally worse than MEC on shapes from the MPEG7-CE dataset, and Figure $4 \mathrm{c}$ indicates that several shapes from the Flavia leaves dataset posed a challenge for both multiscale descriptors, as confirmed in Table 1. It is worth noting that the patterns of the distance matrices reinforce the conclusion that the characterisation of shapes from MPEG7-CE and Flavia leaves is a challenging task for both NMBE and MEC, since the darker square areas outside the main diagonal were larger than expected. 
Table 1. Results of precision $(P)$, recall $(R)$ and F1-score for the cross-validation tests with NMBE, MEC and MONO for the Kimia99, MPEG7-CE and Flavia leaves datasets.

\begin{tabular}{|c|c|c|c|c|c|c|c|c|c|}
\hline \multirow[b]{2}{*}{ Dataset } & \multicolumn{3}{|c|}{$P$} & \multicolumn{3}{|c|}{$R$} & \multicolumn{3}{|c|}{ F1-score } \\
\hline & NMBE & MEC & MONO & NMBE & MEC & MONO & NMBE & MEC & MONO \\
\hline Kimia99 & $78.33 \pm 9.46$ & $78.37 \pm 8.31$ & $82.26 \pm 8.76$ & $75.47 \pm 6.75$ & $72.67 \pm 6.62$ & $76.99 \pm 6.20$ & $74.78 \pm 8.53$ & $72.45 \pm 8.26$ & $77.57 \pm 6.89$ \\
\hline MPEG7 & $62.67 \pm 2.48$ & $66.01 \pm 2.60$ & $63.08 \pm 2.56$ & $60.38 \pm 2.10$ & $63.05 \pm 2.33$ & $59.87 \pm 1.92$ & $58.55 \pm 2.25$ & $61.61 \pm 2.56$ & $57.83 \pm 2.18$ \\
\hline Flavia & $73.48 \pm 1.65$ & $76.17 \pm 1.50$ & $55.83 \pm 1.89$ & $72.60 \pm 1.47$ & $74.82 \pm 1.55$ & $52.81 \pm 1.53$ & $72.09 \pm 1.56$ & $74.63 \pm 1.44$ & $52.56 \pm 1.79$ \\
\hline
\end{tabular}
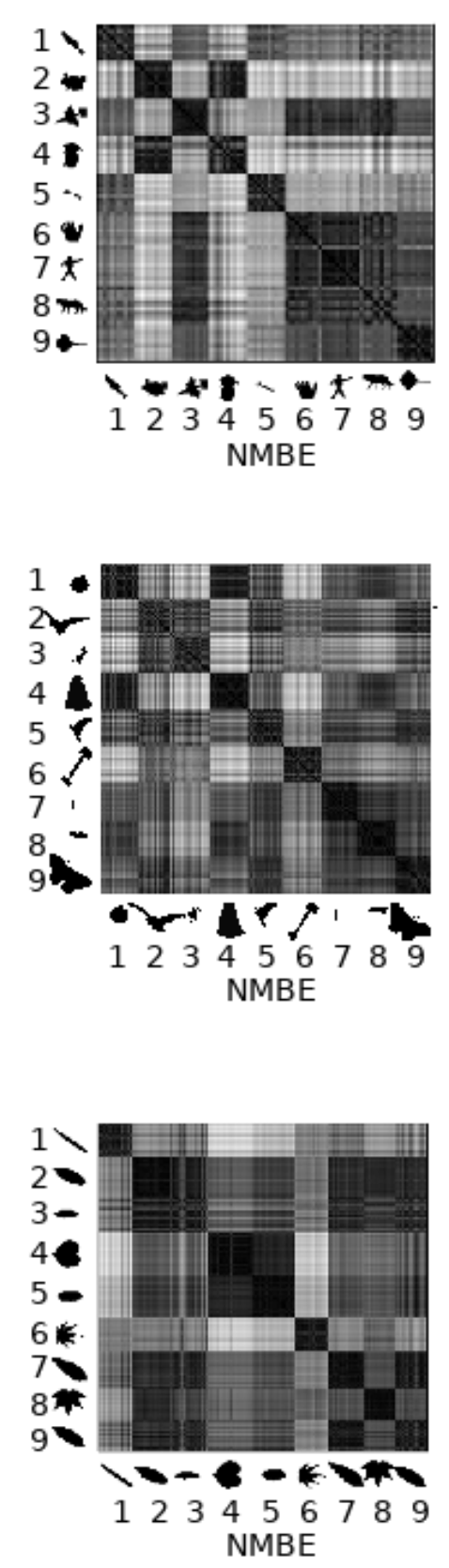

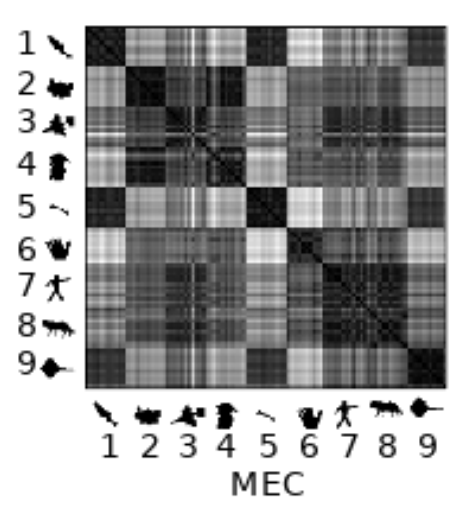

(a)

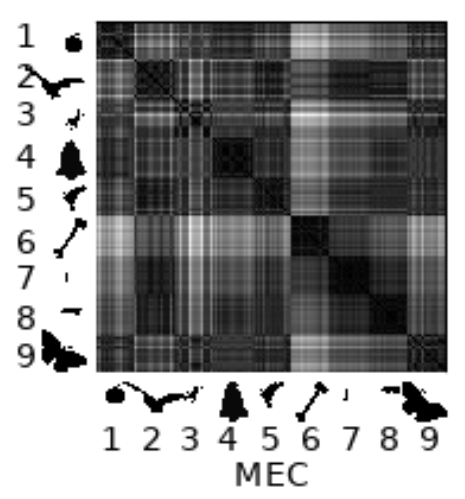

(b)

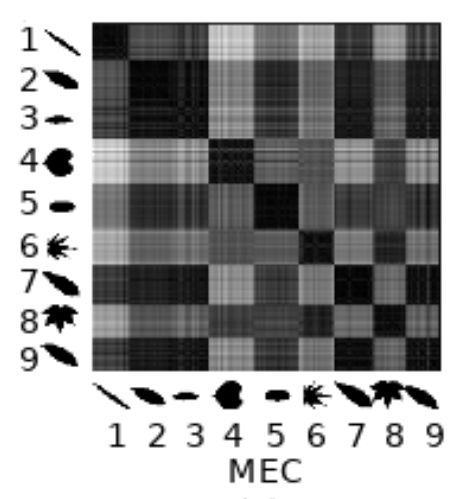

(c)
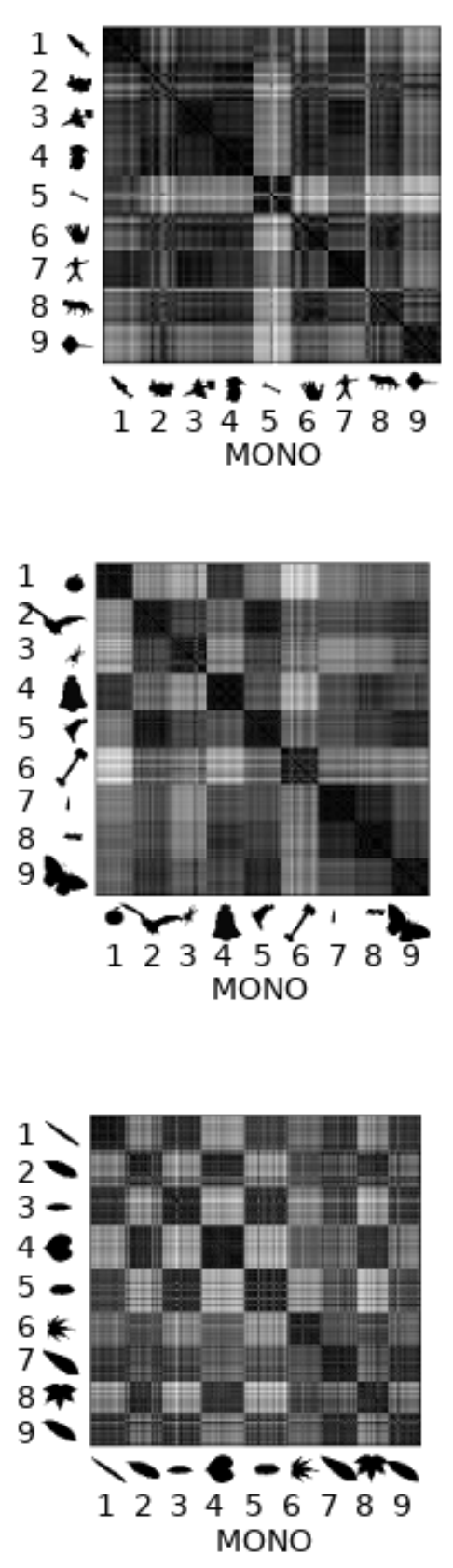

Figure 4. Distance matrices obtained with NMBE, MEC and MONO for the (a) Kimia99; (b) MPEG7-CE; and (c) Flavia leaves datasets. 


\section{CONCLUSION}

In this paper, we propose a multiscale shape descriptor that relies on the differential entropy and curvature, and which is invariant to rotation, translation and scale. Our results show that MEC is relatively sensitive and is a competitive descriptor that can reveal the local features of shapes drawn from three public datasets. We carried out a quantitative evaluation of the performance of MEC using pairwise distance matrices, and a quantitative assessment of classification experiments with plant leaves for a shape recognition task. The results showed that MEC was able to characterise shapes from the MPEG7-CE and Flavia leaves datasets better than NMBE and MONO. Both multiscale shape descriptors performed similarly for shapes from the Kimia99 dataset.

\section{ACKNOWLEDGMENTS}

We are grateful to CNPq (\#306600/2016 - 1), FUNCAP and CAPES-Brazil (Finance Code 001) for partial financial support.

\section{REFERENCES}

Carneiro, A.C., Lopes, J.G.F., Neto, J.F.S.R., Souza, M.M.S., Medeiros, F.N.S., and Bezerra, F.N. (2017). On the evaluation of cost functions for parameter optimization of a multiscale shape descriptor. In 2017 IEEE International Symposium on Signal Processing and Information Technology (ISSPIT), 045-050.

Carneiro, A.C., Lopes, J.G., Souza, M.M.S., Rocha Neto, J.F., Araújo, F.H.D., Silva, R.R.V., Medeiros, F.N., and Bezerra, F.N. (2019). Parameter optimization of a multiscale descriptor for shape analysis on healthcare image datasets. Pattern Recognition Letters, 125, 694-700.

Cesar Jr., R.M. and Costa, L.F. (1996). Towards effective planar shape representation with multiscale digital curvature analysis based on signal processing techniques. Pattern Recognition, 29(9), 1559-1569.

Cesar Jr, R.M. and Costa, L.F. (1997). Application and assessment of multiscale bending energy for morphometric characterization of neural cells. Review of Scientific Instruments, 68(5), 2177-2186.

Costa, L.F. and Cesar Jr, R.M. (2009). Shape Classification and Analysis: Theory and Practice. CRC Press Taylor \& Francis Group, Boca Raton, FL, USA, 2 edition.

Cover, T.M. and Thomas, J.A. (2006). Elements of Information Theory. John Wiley \& Sons, Inc.,Publication, Hoboken, New Jersey, second edition.

Das, V., Dandapat, S., and Bora, P.K. (2019). A novel diagnostic information based framework for superresolution of retinal fundus images. Computerized Medical Imaging and Graphics, 72, 22-33.

Escolano, F., Suau, P., and Bonev, B. (2009). Information Theory in Computer Vision. Springer, London, second edition.

Hasanbelliu, E., Sanchez Giraldo, L., and Principe, J. (2014). Information theoretic shape matching. Pattern Analysis and Machine Intelligence, IEEE Transactions on, 36(12), 2436-2451.

Liu, W., Pokharel, P., and Principe, J. (2006). Correntropy: A localized similarity measure. In IJCNN
'06. International Joint Conference on Neural Networks, 2006., 4919-4924.

Mokhtarian, F. and Mackworth, A. (1992). A theory of multiscale, curvature-based shape representation for planar curves. Pattern Analysis and Machine Intelligence, IEEE Transactions on, 14(8), 789-805.

Page, D., Koschan, A., Sukumar, S., Roui-Abidi, B., and Abidi, M. (2003). Shape analysis algorithm based on information theory. In ICIP 2003. Proceedings. International Conference on Image Processing, 2003., volume 1, I-229-32 vol.1. doi:10.1109/ICIP.2003.1246940.

Paula, Jr., I.C., Medeiros, F.N., Bezerra, F.N., and Ushizima, D.M. (2013). Multiscale corner detection in planar shapes. J. Math. Imaging Vis., 45(3), 251-263. doi: 10.1007/s10851-012-0365-8. URL http://dx.doi.org/ 10.1007/s10851-012-0365-8.

Rényi, A. (1961). On measures of entropy and information. In Proceedings of the Fourth Berkeley Symposium on Mathematical Statistics and Probability, Volume 1: Contributions to the Theory of Statistics, 547-561. University of California Press, Berkeley, Calif.

Silverman, B.W. and Green, P.J. (1986). Density Estimation for Statistics and Data Analysis. Chapman and Hall, London.

Singh, A. and Príncipe, J.C. (2011). Information theoretic learning with adaptive kernels. Signal Processing, 91(2), $203-213$.

Souza, M.M.S., Medeiros, F.N.S., Ramalho, G.L.B., Paula Jr, I.C., and Oliveira, I.N.S. (2016). Evolutionary optimization of a multiscale descriptor for leaf shape analysis. Expert Systems with Applications, 63, 375-385.

Ullman, S. (1995). High-level vision. MIT Press, Cambridge, Massachusetts.

Ushizima, D., Carneiro, A., Souza, M., and Medeiros, F. (2015). Investigating Pill recognition methods for a new National Library of Medicine image dataset, volume 9475, 410-419. Springer.

Webb, A.R. (2002). Statistical Pattern Recognition. John Wiley \& Sons Ltd, 2 edition.

Yang, C. and Yu, Q. (2019). Multiscale fourier descriptor based on triangular features for shape retrieval. Signal Processing: Image Communication, 71, 110-119.

Zhang, D. and Lu, G. (2004). Review of shape representation and description techniques. Pattern recognition, $37(1), 1-19$. 